TRANSACTIONS OF THE

AMERICAN MATHEMATICAL SOCIETY

Volume 188 , Issue 2,1974

\title{
ON PROPER HOMOTOPY THEORY FOR NONCOMPACT 3-MANIFOLDS $\left({ }^{1}\right)$
}

\author{
BY
}

\author{
E. M. BROWN AND T. W. TUCKER
}

\begin{abstract}
Proper homotopy groups analogous to the usual homotopy groups are defined. They are used to prove, modulo the Poincaré conjecture, that a noncompact 3-manifold having the proper homotopy type of a closed product $F \times[0,1]$ or a half-open product $F \times[0,1)$ where $F$ is a 2 -manifold is actually homeomorphic to $F \times[0,1]$ or $F \times[0,1)$, respectively. : By defining a concept for noncompact manifolds similar to boundary-irreducibility, a wellknown result of Waldhausen concerning homotopy and homeomorphism type of compact 3-manifolds is extended to the noncompact case.
\end{abstract}

Introduction. There have been several papers in recent years on noncompact manifolds, proving theorems having homotopy-theoretical hypotheses (particularly "at infinity") and having homeomorphism type conclusions. Some examples are Husch and Price [8], Edwards [6], Siebenmann [12] and [13], Levine et al. [2]. This paper is another of the same.

Siebenmann's paper [12] argues strongly the case that for noncompact spaces the homotopy hypotheses should be on the category of proper maps rather than the category of all continuous maps. We recall that a continuous map $f: X \rightarrow Y$ is proper if $f^{-1}(C)$ is compact whenever $C$ is compact. In this paper we shall work in the category of locally finite simplicial complexes and proper maps, and we shall be concerned with proving theorems about noncompact 3-manifolds.

Our hypotheses will be in terms of functors which are invariants of proper homotopy type (plus a possibly redundant one to get around the 3-dimensional Poincaré conjecture). We remark that a proper homotopy is a homotopy which is proper as a single function of two variables (which is not the same as a continuous family $f_{t}$ of proper maps). In addition to the usual homotopy groups, we shall use two functors which, while invariants of proper homotopy type, are not invariants of the usual (continuous) homotopy type. The first is well known, it is the space

Received by the editors March 17, 1971 and, in revised form, September 20, 1972 and January 22, 1973.

AMS (MOS) subject classifications (1970). Primary 57C99, 57A99, 55A99.

Key words and phrases. Proper map, end, proper homotopy groups, incompressible surface, $p^{2}$-irreducible, boundary-irreducible, end-irreducible.

${ }^{1}$ ) This research was supported in part by NSF Grant GP-16820. 
of ends (cf. Freudenthal [7]), although our approach is somewhat different. The second functor we believe to be new. It is a functor to the category of groups which plays for an end of a noncompact space a role analogous to that played by the fundamental group for a component of a space.

We prove three main theorems. The first is a closed product theorem; it gives necessary and sufficient conditions that a 3-manifold be homeomorphic to $F \times I$, where $F$ is a noncompact 2-manifold. The second theorem is an open collar theorem; it gives necessary and sufficient conditions that a 3-manifold be homeomorphic to $F \times[0, \infty)$ where $F$ is any 2 -manifold other than the projective plane. In [8] this theorem was already proved for compact $F$, but with somewhat different hypotheses. See also [13].

The third theorem is the one with the most applications. In [15] Waldhausen showed that among compact, orientable, irreducible, boundary-irreducible and sufficiently large 3-manifolds, the group system consisting of the fundamental group of the manifold together with the conjugacy classes of the subgroups which are fundamental groups of boundary components characterizes the manifold up to homeomorphism. In [9] Heil extended Waldhausen's theorem to the nonorientable case. For this Heil assumed in addition to Waldhausen's other hypotheses that the manifold contained no 2-sided projective plane $P^{2}$. (As far as we know, no one can yet characterize $P^{2} \times I$ up to homeomorphism.) Our third theorem extends Waldhausen's and Heil's theorems to the noncompact case. In addition to their hypotheses we assume that each component of the boundary is compact, and a condition we call endirreducible which plays for ends the same role that boundary irreducible plays for boundary components. (Indeed if an end arises by throwing away a compact boundary component, then the end is end-irreducible iff the boundary component was boundary irreducible.)

One interesting application of this theorem is to $S^{3}-k$, where $k$ is a knot in the 3-sphere $S^{3}$. If the unique end of $S^{3}-k$ is end-irreducible then we can characterize $s^{3}-k$, up to homeomorphism, by algebraic invariants. In particular this is true of all tame knots (which really follows from Waldhausen's theorem), but it is also true of an uncountable family of wild knots. In fact we do not know of a knot $k$ for which $S^{3}-k$ fails to be end-irreducible.

1 Notation and definitions.

(1.1) Notation. We shall work in the category of locally finite simplicial complexes and piecewise linear ( $\mathrm{pl}$ ) maps. We shall not make a notational distinction between a complex and its underlying topological space. Since we shall work almost entirely in 3 dimensions it follows from [3] that this leads to no trouble. Thus a map is a piecewise linear function, and a proper map is a map such that the counterimage of each compact set is compact.

A manifold will mean a combinatorial manifold. In dimension at most three it 
follows from [11] or [1] that this restriction is no more than paracompactness. If $M$ is a manifold we use $\partial M$ to denote the manifold boundary of $M$. It follows that $\partial(\partial M)$ is empty. If $f: M \rightarrow N$ is a map of manifolds, then the statement " $f$ is proper" has two meanings. Zeeman [17] and many authors following him have used it to mean $f^{-1}(\partial N)=\partial M$. If we speak of a map between manifolds as proper we shall mean it in this second sense only when the domain is compact (especially the unit interval $I$ or the 1 -sphere $S^{1}$ ). If $M$ is a manifold, and $A \subset M$ is a finite polyhedron, then a regular neighborbood $U(A)$ is meant in the sense of $\mathrm{J}$. H. C. Whitehead [16] (see also [17]). However we shall also require that $U(A) \cap$ $\partial M$ is a regular neighborhood of $A \cap \partial M$ in $\partial M$.

If $K$ is a complex and $A \subset K$ is a subset then $\mathrm{cl}(A)$ is the closure of $A$ in $K$, and $\operatorname{Fr}(A)$ is the frontier of $A$ in $K$ (sometimes called the boundary, but we avoid that terminology). We say (following [14]) that $A$ is unbounded (in $K)$ if $\operatorname{cl}(A)$ is not compact.

The letters $m$ and $n$ denote variables ranging over the nonnegative integers, while $s$ and $t$ range over the nonnegative real numbers. On occasion these letters denote particular values, but only when the context makes this clear. Otherwise we use a subscript, especially zero. We use the notation $n \gg 0$ (or $t \gg 0$ ) to mean "for all $n$ (or $t$ ) sufficiently large". This usually occurs after a statement involving $n$ which is only asserted for $n$ greater than some fixed, but unspecified, value $n_{0}$.

(1.2) Ends. Let $K$ be a complex. Proper maps $b, b^{\prime}:[0, \infty) \rightarrow K$ are said to determine the same end (of $K$ ) provided for $C \subset K$ compact and $t \gg 0, b(t)$ and $b^{\prime}(t)$ are in the same component of $K-C$. This is clearly an equivalence relation, the equivalence class of $b$ is denoted [b] and is called an end of $K$. The set of ends is denoted $\underline{\pi}_{0}(K)$ and is topologized as follows: If $C$ is as above and $A$ is an unbounded component of $K-C$, then the set of ends $[b]$ so that $b(t) \in A$ for $t \gg 0$ is declared to be an open set. We refer to it as the open set determined by $A$ and denote it by $\underline{A}$. The space $\underline{\pi}_{0}(K)$ is 0-dimensional and Hausdorff. The components of $K$ partition $\underline{\pi}_{0}(K)$ into open, compact, and second countable subspaces (i.e. compact subset of the cantor set).

A sequence $\left\{U_{n}\right\}$ of subsets of $K$ is said to converge to the end [b] of $K$ provided for any compact $C \subset K$ and component $A$ of $K-C$ with $[b] \in \underline{A}$ we have $U_{n} \subset A$ for $n \gg 0$. If $U_{n} \neq \varnothing$ for $n \gg 0$ then $[b]$ is unique.

(1.3) Germs and proper homotopies. Let $K$ and $L$ be complexes, and let $f$, $f^{\prime}: K \rightarrow L$ be proper maps. We say $f$ and $f^{\prime}$ have the same germ (at infinity) if for some compact set $C \subset K, f(x)=f^{\prime}(x)$ when $x \in K-C$. This is an equivalence relation, the equivalence class of $f$ is denoted $f$, and is called the germ of $f$.

Notice if $b, b^{\prime}:[0, \infty) \rightarrow t$ are proper, and $\underline{b}=\underline{b}^{\prime}$, then $[b]=\left[b^{\prime}\right]$. 
If $f, f^{\prime}: K \rightarrow L$ are proper maps, a proper bomotopy from $f$ to $f^{\prime}$ is a proper map $H: K \times I \rightarrow L$ with $H(x, 0)=f(x)$ and $H(x, 1)=f^{\prime}(x)$. If $H$ exists, $f$ and $f^{\prime}$ are said to be proper homotopic; this is an equivalence relation on the set of proper maps from $K$ to $L$. If $J \subset K$ we say $f$ and $f^{\prime}$ are proper bomotopic rel $J$ if $H$ may be chosen constant on $J$, that is, $H(x, t)=f(x)$ for all $(x, t) \epsilon J \times I$.

Germs $f$ and $f^{\prime}$ are said to be proper homotopic if they have proper homotopic representatives. Similarly for proper homotopic rel $\mathrm{J}$.

Complexes $K$ and $L$ are said to have the same proper bomotopy type if there exist proper maps $f: K \rightarrow L$ and $g: L \rightarrow K$ so that $f g$ and $g b$ are proper homotopic to the respective identity maps.

If $f: K \rightarrow L$ is a proper map, then $f$ induces (by composition) a continuous map $\underline{\pi}_{0}(f): \underline{\pi}_{0}(K) \rightarrow \underline{\pi}_{0}(L)$. If $f$ and $f^{\prime}$ are proper homotopic then $\underline{\pi}_{0}(f)=\underline{\pi}_{0}\left(f^{\prime}\right)$. Thus, for example, the identity map of the real line $R^{1}$ on itself is not proper homotopic to the map $x \rightarrow-x$.

(1.4) $\pi_{1}$. We denote by $\underline{S}^{1}$ the complex consisting of the ray $[0, \infty)$ together with a different copy of the 1-sphere $S^{1}$ attached at each integer point. The sub-

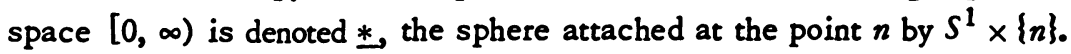

Let $K$ be a complex, [a] an end of $K$, and let $a$ be representative of [a]. By a proper map $\lambda:\left(S^{1}, *\right) \rightarrow(K, \underline{a})$, we mean a proper map $\lambda: \underline{S}^{1} \rightarrow K$ with the germ of $\lambda \mid \underline{*}$ equal to $\underline{a}$. On the set of germs of such maps, consider the relation of proper homotopy rel $\underline{*}$. This is an equivalence relation. The equivalence class of $\lambda$ is denoted $[\lambda]$. The set of equivalence classes is denoted $\underline{\pi}_{1}(K, \underline{a})$; we introduce a group structure. Let $\lambda$ and $\mu$ represent classes $[\lambda]$ and $[\mu]$. Choose $n_{0}$ so that for $t \geq n_{0}, \lambda(t)=\mu(t)$. Let $\theta$ be the angular parameter on $S^{1}$ and define $\nu: S^{1} \times\{n\} \rightarrow K$ for $n \geq n_{0}$ by:

$$
\nu(\theta, n)= \begin{cases}\lambda(2 \theta, n), & 0 \leq \theta \leq \pi, \\ \mu(2 \theta-2 \pi, n), & \pi \leq \theta \leq 2 \pi .\end{cases}
$$

Let $\nu(t)=\lambda(t)$ for $t \geq n_{0}$, and extend $\nu$ over $\underline{S}^{1}$ to be continuous. Then $\nu$ is a $\operatorname{proper} \operatorname{map}\left(\underline{S}^{1}, \underline{*}\right) \rightarrow(K, \underline{a})$ and $[\nu]=[\lambda] \cdot[\mu]$.

The proofs used in the construction of the fundamental group of a complex carry over quite easily to show that $[\nu]$ depends only on $[\lambda]$ and $[\mu]$, and that with this multiplication $\pi_{1}(K, \underline{a})$ is a group. If $f: K \rightarrow L$ is a proper map of complexes then $f$ induces (by composition) a function $\underline{\pi}_{1}(f): \underline{\pi}_{1}(K, \underline{a}) \rightarrow_{\underline{\pi}_{1}}(L, L a)$. Again the usual proofs easily lead to proofs that $\underline{n}_{1}(f)$ is a homomorphism, that $\underline{m}_{1}$ is a functor to the category of groups, and that if $f, f^{\prime}: K \rightarrow L$ are proper homotopic rel $\underline{a}$, then $\underline{\pi}_{1}(f)=\underline{\pi}_{1}\left(f^{\prime}\right)$.

We call $\underline{\pi}_{1}(K, \underline{a})$ the proper fundamental group of the end $[a]$ of $K$ based on $\underline{a}$. 
That both the name and the functor are reasonable is shown in [4], where this functor and its higher order analogues are investigated. In particular a Whiteheadtype theorem is proved showing that if a proper map induces isomorphisms of all the usual homotopy groups and all the proper homotopy groups, then it is a proper homotopy equivalence. Moreover a functor is given to compute $\underline{\pi}_{1}(K, \underline{a})$ in terms of the usual fundamental groups of certain unbounded subsets (and also for the higher order analogues).

If, in the above definition, we delete the word proper, that is, we consider homotopy classes rel $\underline{*}$ of germs of maps $\left(S^{1}, \underline{*}\right) \rightarrow(K, \underline{a})$ we again get a group (same addition) denoted $\pi_{1}(K, a)$. We call this the repeated fundamental group of the end $[a]$ of $K$ based at $\underline{a}$. Clearly a map $f: K \rightarrow L$ induces a homomorphism $\pi_{1}(f): \pi_{1}(K, \underline{a}) \rightarrow \pi_{1}(L, \underline{f a})$, and with this definition $\pi_{1}$ is a functor to the category of groups.

Suppose again that $K$ is a complex, $a, b:[0, \infty) \rightarrow K$ are proper maps representing germs $\underline{a}$ and $\underline{b}$, and that $[a]=[b]$. A patb in $K$ from $\underline{a}$ to $\underline{b}$ is a sequence of maps $\beta_{n}: I \rightarrow K$ so that $\beta_{n}(l)$ converges to $[a]$, and $\beta_{n}(0)=a(n), \beta_{n}(1)=b(n)$ for $n \gg 0$. The condition $[a]=[b]$ implies the existence of such a path. A given path induces an isomorphism $\underline{\pi}_{1}(K, \underline{a}) \rightarrow \underline{\pi}_{1}(K, \underline{b})$. Indeed if $\lambda$ represents an element of $\underline{\pi}_{1}(K, \underline{a})$ then we switch the base point of each $\lambda \mid s^{1} \times\{n\}$ along $\beta_{n}$ to $b(n)$. This, together with $b$, gives us a map $\left(\underline{S}^{1}, \underline{*}\right) \rightarrow(K, \underline{b})$ which is proper since $\left\{\beta_{n}(l)\right\}$ converges to $[a]$.

Similarly $\left\{\beta_{n}\right\}$ induces an isomorphism $\pi_{1}(K, \underline{a}) \rightarrow \pi_{1}(K, \underline{b})$. The former isomorphism depends (at most) on the proper homotopy class rel end points of the germ of $\left\{\beta_{n}\right\}$, the latter isomorphism on the homotopy class.

We note for later use: If $K$ and $L$ are complexes, $a:[0, \infty) \rightarrow K$ and $b:[0, \infty) \rightarrow$ $L$ are maps, and $\psi_{n}: \pi_{1}(K, a(n)) \rightarrow \pi_{1}(L, b(n))$ is a homomorphism for each $n$, then $\left\{\psi_{n}\right\}$ induces a homomorphism $\psi^{\infty}: \pi_{1}(K, \underline{a}) \rightarrow \pi_{1}(L, \underline{b})$.

(1.5) Manifolds. We follow much of the terminology of [9] and [15] here. Thus a 3-manifold $M$ is irreducible if every (pl) embedded 2-sphere in $M$ bounds a ball. $M$ is $P^{2}$-irreducible if $M$ is irreducible and contains no 2 -sided projective planes.

If $F$ is a 2-manifold properly embedded in $M$, we say $F$ is incompressible in $M$ provided $F \cap \partial M=\partial F, F$ is 2-sided in $M$, no component of $F$ is a 2-sphere bounding a ball in $M$, and if $D$ is a disk in $M$ with $D \cap F=\partial D$ then $\partial D$ bounds a disk in $F$. It is a consequence of Dehn's lemma and the loop theorem that this last condition is equivalent to $\pi_{1}(G) \rightarrow \pi_{1}(M)$ is a monomorphism, where $G$ is any component of $F$ and we are using the inclusion induced homomorphism.

If $M$ is a 3 -manifold we say $M$ is boundary irreducible if $\partial M$ is incompressible in $M$. We say $M$ is end-irreducible if $M \neq R^{3}$ and for each end [a] of $M$, the homomorphism $\underline{\pi}_{1}(M, \underline{a}) \rightarrow \pi_{1}(M, \underline{a})$ is a monomorphism. By the above this is independent of the choice of $\underline{a}$ representing $[a]$. 
(1.6) Exbausting sequences. Let $K$ be a countable complex (e.g. let $K$ be connected). An exbausting sequence for $K$ is a sequence $\left\{C_{n}\right\}$ of compact sets so that $C_{n}$ is contained in the interior of $C_{n+1}$, and $\bigcup_{n} C_{n}=K$. If $\left\{C_{n}\right\}$ is an exhausting sequence then so is any subsequence. We shall frequently have to choose a subsequence of an exhausting sequence with some nice property. Having shown that the subsequence exists, we shall usually then proceed as if the original sequence had the property. Cf. Waldhausen's remarks in [15] about "induction on niceness".

Since we have assumed $K$ countable, exhausting sequences for $K$ always exist. Indeed we may choose an exhausting sequence whose terms are subcomplexes of $K$. If $K$ is connected we may choose the terms to be connected.

If $K$ is connected and $\left\{C_{n}\right\}$ is an exhausting sequence for $K$, then the unbounded components of $K-C_{n}$ determine a finite open cover $U_{n}$ of $\underline{\pi}_{0}(K)$. (This is not hard, check it first for an exhausting sequence of subcomplexes, and then use that each term of one exhausting sequence is eventually contained in a term of another.) $U_{n+1}$ is a partition of $U_{n}$, and $U_{n} U_{n}$ is a basis for the topology of $\underline{\pi}_{0}(K)$.

2. The closed product theorem.

(2.1) Lemma. Let $M$ be a compact, connected, $P^{2}$-irreducible 3-manifold. Let $F \subset \partial M$ be a compact, connected 2-manifold with $\pi_{1}(F) \rightarrow \pi_{1}(M)$ an isomorpbism. Then $M$ is bomeomorphic to $F \times I$ with $F$ corresponding to $F \times\{0\}$.

Proof. If $F$ is a disk, then $M$ is a ball and we are done. Assume $F$ is not a disk then $\pi_{1}(F)$ is infinite, hence $\pi_{1}(M)$ is infinite. By the usual argument $P^{2}$. irreducible implies $M$ is aspherical, so the inclusion map $F \hookrightarrow M$ is a homotopy equivalence. It follows that $\partial M \neq F$ (otherwise $H_{2}\left(F ; Z_{2}\right) \rightarrow H_{2}\left(M ; Z_{2}\right)$ is trivial). Then by $[5$, Theorem (3.4)] the lemma follows.

(2.2) Theorem. Let $M$ be a connected, noncompact $P^{2}$-irreducible 3-manifold. Let $F \subset \partial M$ be a connected 2-manifold with $\partial M-F \neq \varnothing$. Let $i: F \subset M$ be the inclusion map and suppose

(a) $\pi_{1}(i): \pi_{1}(F) \rightarrow \pi_{1}(M)$ is an isomorphism,

(b) $\underline{\pi}_{0}(i): \underline{\pi}_{0}(F) \rightarrow \underline{\pi}_{0}(M)$ is a bomeomorpbism,

(c) $\underline{\pi}_{1}(i): \underline{\pi}_{1}(F, \underline{a}) \rightarrow \underline{\pi}_{1}(M, \underline{a})$ is an epimorphism,

for each end $[a]$ of $M$. Then $M$ is bomeomorpbic to $F \times I$ with $F$ going to $F \times\{0\}$.

Proof. As above it follows that $i: F \hookrightarrow M$ is a homotopy equivalence. Let us denote one component of $\operatorname{cl}(\partial M-F)$ by $G \cup(\partial G \times l)$ where we have collared the boundary so that $\partial G \times\{1\}$ corresponds to $\partial G$. We claim that $F$ noncompact and $H_{2}\left(M, F ; Z_{2}\right)=0$ implies $G$ is noncompact. Otherwise $G \cup(\partial G \times I)$ is a 
cycle of $C_{2}\left(M, F ; Z_{2}\right)$ since $\partial(G \cup(\partial G \times I)) \subset \partial F$. Then $H_{2}\left(M, F ; Z_{2}\right)=0$ implies that $G \cup(\partial G \times I)$ is $Z_{2}$-homologous modulo its boundary to a finite $Z_{2}$-chain $F_{1}$ of $F$. But $\partial^{2}(G \cup(\partial G \times I))=0$ implies $F_{1}$ is a submanifold of $F$ with $\partial G=F_{1} \cap$ $\partial F$. Then $F$ connected implies $F_{1}=F$, but $F_{1}$ is compact and $F$ is not. Let us choose an exhausting sequence $\left\{G_{n}\right\}$ for $G$ so that

(i) $G_{n}$ is a connected polyhedral 2-manifold in $G$;

(ii) the frontier $\operatorname{Fr}\left(G_{n}\right)$ of $G_{n}$ in $G$ consists of arcs and simple closed curves all properly imbedded in $G$;

(iii) components of $G-G_{n}$ are all unbounded (and hence finite in number). This is possible since $G$ is connected (cf. (3.1)).

If $\lambda$ is a component of $\operatorname{Fr}\left(G_{n}\right)$ which is a simple closed curve, then $\lambda$ is freely homotopic to a loop in $F$ since $\pi_{1}(F) \rightarrow \pi_{1}(M)$ is an epimorphism. It follows from [5, Lemma (2.3)] that in an arbitrarily small neighborhood of the image of such a free homotopy there is a proper embedding of $\lambda \times I$ with $\lambda \times\{1\}$ going to $\lambda$, and $\lambda \times\{0\}$ going properly into $F$. Similarly, if $a$ is a component of $\operatorname{Fr}\left(G_{n}\right)$ which is an arc, then $a \cup(\partial \alpha) \times I$ is homotopic with endpoints fixed to a path in $F$. Again, by [5, Lemma (2.3)], in any neighborhood of the image of such a homotopy there is a proper embedding of $\alpha \times I$ with $\alpha \times\{1\} \cup(\partial \alpha) \times I$ going to $\alpha \cup(\partial \alpha) \times I$ and with $a \times\{0\}$ being embedded in $F$.

Given embeddings as above of the components of $\operatorname{Fr}\left(G_{n}\right) \times I$, we may assume them all to be in general position with respect to one another by a small deformation. By standard cut and paste arguments we may remove all the intersection curves staying in any small neighborhood of the union. This process need not change $\operatorname{Fr}\left(G_{n}\right) \times\{1\} \cup\left(\partial \operatorname{Fr}\left(G_{n}\right)\right) \times I$. Thus given any homotopy of $\operatorname{Fr}\left(G_{n}\right) \cup$ $\left(\partial \operatorname{Fr}\left(G_{n}\right)\right) \times I$ into $F$ with endpoints fixed, in any small neighborhood of the image we can find a proper embedding of $\operatorname{Fr}\left(G_{n}\right) \times l$ with $\operatorname{Fr}\left(G_{n}\right) \times\{1\}$ going to $\operatorname{Fr}\left(G_{n}\right)$, $\partial \operatorname{Fr}\left(G_{n}\right) \times I$ going by the identity and $\operatorname{Fr}\left(G_{n}\right) \times\{0\}$ going properly into $F$.

Let $\left\{C_{m}\right\}$ be an exhausting sequence for $M$. For each $n$ choose an embedding as above of $\operatorname{Fr}\left(G_{n}\right) \times I$ into $M$ so as to miss as many of the sets $C_{m}$ as possible. We claim that the resulting map of $\bigcup_{n} \operatorname{Fr}\left(G_{n}\right) \times I$ into $M$ is a proper map. Since each embedding may be constructed in an arbitrarily small neighborhood of a homotopy, it is sufficient to show: For each $m_{0}$ if $n \gg 0$ there is a homotopy of $\operatorname{Fr}\left(G_{n}\right) \cup\left(\partial \operatorname{Fr}\left(G_{n}\right)\right) \times I$ rel $\partial \operatorname{Fr}\left(G_{n}\right) \times\{0\}$, into $F$ whose image misses $C_{m_{0}{ }^{\circ}}$ If this is not true then either:

(1) there is a sequence $\left\{\lambda_{n}\right\}$ of loops in $G$ converging to an end [b] of $M$, and an integer $m_{0}$ so that the image of any free homotopy of $\lambda_{n}$ into $F$ meets $C_{m_{0}}$, or

(2) there is a sequence $\left\{a_{n}\right\}$ of proper arcs in $G$ converging to an end $[b]$ of $M$, and an integer $m_{0}$, so that the image of any homotopy of $\alpha_{n} \cup\left(\partial \alpha_{n}\right) \times I$ rel endpoints into $F$ meets $C_{m_{0}}$. We shall derive a contradiction for each case. 
Suppose (1) holds. Choose a representative $b$ of $[b]$ so that $b(n)$ is a chosen base point of $\lambda_{n}$. Let $a$ represent an element $[a]$ of $\underline{\pi}_{0}(F)$ with $\underline{\pi}_{0}(i)([a])=[b]$. Choose a proper path $\left\{\beta_{n}\right\}$ from $a$ to $b$ (see (1.4)). Let $[\lambda]$ be the element of $\underline{\pi}_{1}(M, \underline{b})$ defined by $b$ and the $\lambda_{n}$. Let $[\mu]$ be the element of $\underline{\pi}_{1}(M, \underline{a})$ which the isomorphism induced by $\left\{\beta_{n}\right\}$ carries to $[\lambda]$. Since $\underline{I}_{1}(i)$ is an epimorphism, some representative $\mu$ of $[\mu]$ lies in $F$. For $n \gg 0$ this provides a free homotopy of $\lambda_{n}$ into $F$ missing $C_{m 0^{\circ}}$

Suppose (2) holds. Since $\underline{\pi}_{0}(i)$ is one-to-one there is a sequence $\beta_{n}$ of paths in $F$ converging to $[b]$, and joining the points $\left(\partial \alpha_{n}\right) \times\{0\}$. Let $b$ be a representative of $[b]$ whose image lies in $F$, and so that $b(n) \in\left(\partial \alpha_{n}\right) \times\{0\}$. Then $b$, together with the loops formed by $\beta_{n} \cup\left(\partial a_{n}\right) \times l$, gives an element of $\underline{\pi}_{1}(M, \underline{b})$. Again since $\underline{\pi}_{1}(i)$ is an epimorphism some representative of this element lies in $F$. Again for $n \gg 0$ this provides a homotopy rel endpoints of $\alpha_{n} \cup\left(\partial \alpha_{n}\right) \times I$ into $F$ whose image misses $C_{m 0^{\circ}}$.

This establishes the fact that our map is proper. It then follows that by choosing a subsequence of $\left\{G_{n}\right\}$ we may assume that our map $\bigcup_{n} \operatorname{Fr}\left(G_{n}\right) \times I \rightarrow M$ is an embedding. We identify $\bigcup_{n} \operatorname{Fr}\left(G_{n}\right) \times I$ with its image in $M$, which we note is consistent with $(\partial G) \times I$.

We next claim that there is a compact connected 3-manifold $M_{n}$ in $M$ with $\operatorname{Fr}\left(M_{n}\right)=\operatorname{Fr}\left(G_{n}\right) \times I$, and $M_{n} \cap G=G_{n} .\left(^{2}\right)$ Indeed $G_{n} \cup \partial\left(G_{n}\right) \times I$ is a $Z_{2}$ 2-cycle of $M \bmod F$. As before $H_{2}\left(M, F ; Z_{2}\right)=0$, so it is a $Z_{2}$-boundary as well, and $M_{n}$ is a 3-chain which it bounds. $M_{n}$ is connected since $G_{n}$ is connected, and is compact since it is a finite $Z_{2}$-chain. We let $F_{n}=M_{n} \cap F$, then $\partial M_{n}=F_{n} \cup G_{n} \cup$ $\left(\partial G_{n}\right) \times I$, and $\operatorname{Fr}\left(F_{n}\right)=\operatorname{Fr}\left(G_{n}\right) \times\{0\}$.

We claim that $F_{n}$ is connected. If not, there is a path in $M_{n}$ joining interior points of distinct components of $F_{n}$. Such a path has $Z_{2}$ intersection number zero with each component of $\operatorname{Fr}\left(G_{n}\right) \times I$. We homotope this path rel endpoints into $F$. The resulting path has $Z_{2}$ intersection number zero with each component of $\operatorname{Fr}\left(F_{n}\right)$, contradicting the fact that it joins interior points of distinct components of $F_{n}$.

We now claim that the inclusion induced homomorphism $\pi_{1}\left(F_{n}\right) \rightarrow \pi_{1}\left(M_{n}\right)$ is an isomorphism. Suppose first that there is a disk $D$ which is the closure of a component of $F-\partial F_{n^{*}}$. Then $\partial D \subset\left[\partial G \cup \operatorname{Fr}\left(G_{n}\right)\right] \times\{0\}$ so $(\partial D) \times I$ is contained in $\left[\partial G \cup \operatorname{Fr}\left(G_{n}\right)\right] \times I$. Now $(\partial D) \times I$ separates $M$ because $\partial D$ separates $F$, and the component of $M-(\partial D) \times I$ meeting $F$ in the interior of $D$ must be bounded since $\underline{\pi}_{0}(i)$ maps $\underline{\pi}_{0}(F)$ onto $\underline{\pi}_{0}(M)$. Then the closure of this component is $M_{n}$ since components of $G-G_{n}$ are unbounded. Using a regular neighborhood of $F_{n}$ in $M_{n}$

\footnotetext{
$\left({ }^{2}\right)$ We are indebted to Dennis Sullivan for this argument.
} 
we may move $M_{n}$ up off $F$ to $M_{n}^{\prime}$, still homeomorphic to $M_{n}$ but so that $M-M_{n}^{\prime}=$ $\left(M-M_{n}\right) \cup\left(F_{n} \times[0, \epsilon)\right)$. Let $F_{n}^{\prime}=F_{n} \times\{\epsilon\} \subset \partial M_{n}^{\prime}$. Using $*$ to denote free product of groups we have $\pi_{1}(M)=\pi_{1}\left(\operatorname{cl}\left(M-M_{n}^{\prime}\right)\right) * \pi_{1}\left(M_{n}^{\prime}\right)$. Since $F \subset M-M_{n}^{\prime}$ it follows that $\pi_{1}\left(M_{n}^{\prime}\right)=1, G_{n}$ is a disk, and $M_{n}$ is a ball. In this case $\pi_{1}\left(F_{n}^{\prime}\right) \rightarrow \pi_{1}\left(M_{n}\right)$ is an isomorphism.

On the other hand suppose no such disk exists. Letting $M_{n}^{\prime}$ and $F_{n}^{\prime}$ be as above it follows that $\pi_{1}\left(F_{n}^{\prime}\right) \rightarrow \pi_{1}\left(M_{n}^{\prime}\right)$ and $\pi_{1}\left(F_{n}^{\prime}\right) \rightarrow \pi_{1}\left(\operatorname{cl}\left(M-M_{n}^{\prime}\right)\right)$ are monomorphisms (both inclusion induced). Then $\pi_{1}(M)$ is the free product of $\pi_{1}\left(\operatorname{cl}\left(M-M_{n}^{\prime}\right)\right)$ by $\pi_{1}\left(M_{n}^{\prime}\right)$ with amalgamation over $\pi_{1}\left(F_{n}^{\prime} \cup\left(F_{r}\left(F_{n}\right) \times[\epsilon, 1]\right)\right)$. Since $\pi_{1}\left(\operatorname{cl}\left(M-M_{n}^{\prime}\right)\right) \rightarrow \pi_{1}(M)$ is an epimorphism as above, it follows that $\pi_{1}\left(F_{n}^{\prime}\right) \rightarrow$ $\pi_{1}\left(M_{n}\right)$ is an isomorphism (cf. Proposition 2.4 of [18]).

It now follows from (2.1) that $M_{n}$ is homeomorphic to $F_{n} \times I$ so as to preserve $\left(\partial F_{n}\right) \times l$. It follows from [5, Theorems 4.1 and 5.1] that the homeomorphism of $M_{n+1}$ onto $F_{n+1} \times I$ may be chosen to extend the homeomorphism of $M_{n}$ onto $F_{n} \times I$ unless $F_{n}$ is a disk, and $F_{n} \times I$ is knotted in $F_{n+1} \times I$. Assuming for the moment that this last does not happen for all $n \gg 0$, by a subsequence we may assume it never happens. Then the above homeomorphisms fit together to give a homeomorphism of $F \times I$ onto $\bigcup_{n} M_{n}$. Since the map $\bigcup_{n} \operatorname{Fr}\left(G_{n}\right) \times I \rightarrow M$ is proper, and $M$ is connected it follows that $\bigcup_{n} M_{n}=M$ and we are done.

We must only show that for every $n, F_{n}$ a disk and $F_{n} \times I$ knotted in $F_{n+1} \times I$ is impossible. If this happens then $F$ is the Euclidean plane $R^{2}$ (as is $G$ ) and for each $n>1$, there is a loop $\lambda_{n}$ in $F_{n} \times I-F_{n-1} \times I$ which is not freely homotopic to any loop in $F-F_{1}$. The same contradiction reached in (1) above can be reached in this case also.

We make a few remarks on this theorem. One compact version was proved in [5], the form closest to this one is Lemma (2.1) above. The $\underline{\pi}_{0}(i)$ hypotheses are necessary; if we remove only one point of $F \times\{1\}$ from $F \times I$ we only destroy the fact that $\pi_{0}(i)$ is onto. On the other hand if $F=R^{2}-p t$ then $F \times[0,1)$ together with the interior of a closed disk in $F \times\{1\}$ satisfies all but $\underline{\pi}_{0}(i)$ is one-to-one. Finally the last paragraph provides a prescription for constructing 3-manifolds bounded by two planes, which are an increasing union of cells (and hence $P^{2}$. irreducible) and satisfying all hypotheses of the theorem except that $\underline{m}_{1}(i)$ is not an epimorphism.

3. The open product theorem.

(3.1) Lemma. Let $M$ be a connected end-irreducible 3-manifold. There exists an exbausting sequence $\left\{C_{n}\right\}$ for $M$ so that

(i) $C_{n}$ is a compact submanifold of $M$,

(ii) $\operatorname{Fr}\left(C_{n}\right)$ is a system of properly embedded surfaces in M meeting no compact 
component of $\partial M$,

(iii) $\operatorname{Fr}\left(C_{n}\right)$ is incompressible in $M$,

(iv) $C_{n}$ is connected,

(v) all components of $M-C_{n}$ are unbounded.

Proof. Suppose $\left\{C_{n}^{\prime}\right\}$ is a sequence satisfying (i)-(iv). If $C_{n}$ is the union of $C_{n}^{\prime}$ with the bounded components of $M-C_{n}^{\prime}$ then $\left\{C_{n}\right\}$ is an exhausting sequence satisfying (i)-(v). The proof that $C_{n}$ is compact is as in the last paragraph of (1.6). That (iii) still holds for $C_{n}$ follows since a system of surfaces is incompressible iff each component is incompressible. The others are clear, so we shall now construct $\left\{C_{n}^{\prime}\right\}$ satisfying (i)-(iv).

Suppose next that $\left\{C_{n}^{\prime}\right\}$ satisfies (i)-(iii). Define $\left\{C_{n}\right\}$ by the following inductive procedure: Let $C_{1}$ be a component of $C_{1}^{\prime}$. Choose $C_{n}$ to be the component of $C_{n}^{\prime}$ containing $C_{n-1}$. If $C_{n_{0}}^{\prime \prime}$ is a component of $C_{n_{0}}^{\prime^{n}}$ we may join it to $C_{n_{0}}$ by a path, the result is a compact connected set. If $n \gg 0$ this set is contained in the interior of $C_{n}^{\prime}$, since it is connected it is contained in $C_{n}$. It follows that $U_{n} C_{n}=M$. Then $\left\{C_{n}\right\}$ satisfies (i)-(iv).

Suppose $\left\{C_{n}^{\prime}\right\}$ satisfies (i) and (ii). If $\operatorname{Fr}\left(C_{n}^{\prime}\right)$ is compressible in $M$ then some simple loop $\lambda$ which is essential on $\operatorname{Fr}\left(C_{n}^{\prime}\right)$ bounds a properly embedded disk $D$ in either $M-C_{n}^{\prime}$ or in $C_{n}^{\prime}$. In the first case we add a regular neighborhood of $D$ to $C_{n}^{\prime}$, in the second we remove an open regular neighborhood of $D$ from $C_{n}^{\prime}$. This decreases the genus of $\operatorname{Fr}\left(C_{n}^{0}\right)$ if $\lambda$ is a nonseparating loop, and otherwise separates some component of $\operatorname{Fr}\left(C_{n}^{\prime}\right)$ into two components neither having genus zero. Thus the procedure terminates giving $C_{n}$ with $\operatorname{Fr}\left(C_{n}\right)$ incompressible in $M$.

The operation which removes part of $C_{n}^{\prime}$ raises two problems. First we might remove part of $C_{n-1}$ preventing an inductive construction. But if $\operatorname{Fr}\left(C_{n-1}\right)$ is incompressible in $M$ then it is sufficient to make $\operatorname{Fr}\left(C_{n}\right)$ incompressible in $\overline{M-C_{n-1}}$, so we may choose the $D$ 's to miss $C_{n-1}$. The second problem is that after the inductive construction the sequence $\left\{C_{n}\right\}$ may no longer exhaust $M$. It is at this point that we need $M$ to be end-irreducible, indeed, condition (iii) is equivalent to end-irreducible. Each modification of $\operatorname{Fr}\left(C_{n}^{\prime}\right)$ removes an annulus and replaces it with a pair of disks. Choose each $\lambda$ to miss these disks so that each $\lambda$ is contained in $\operatorname{Fr}\left(C_{n}^{\prime}\right)$. Let $\left\{E_{n}\right\}$ be a fixed exhausting sequence for $M$, and having chosen $\lambda$, choose $D$ to miss as many of the sets $E_{n}$ as possible.

We assert that for each integer $k$, there exists an integer $n$ so that $E_{k} C$ int $C_{n}^{\prime}$ and that none of the $D^{\prime}$ 's chosen to modify $C_{n}^{\prime}$ meet $E_{k}$, whence $E_{k} \subset C_{n}$ as well. If this is false for some $k_{0}$ then we produce a sequence $\left\{\lambda_{n}\right\}$ of simple loops, $\lambda_{n} \subset \operatorname{Fr}\left(C_{n}^{\prime}\right), \lambda_{n}$ inessential in $M$, but essential in $M-E_{k_{0}}$. A subsequence converges to some end [a] of $M$ and this gives us a nontrivial element in 
$\operatorname{ker}\left(\pi_{1}(M, \underline{a}) \rightarrow \pi_{1}(M, \underline{a})\right)$. Thus the assertion is true which shows that $\left\{C_{n}\right\}$ exhausts $M$.

The proof will be finished when we show that there is an exhausting sequence satisfying (i) and (ii). This is almost trivial, start with any exhausting sequence $\left\{C_{n}^{\prime}\right\}$ and modify it by including in $C_{n}^{\prime}$ any compact component of $\partial M$ which it meets. Take a second regular neighborhood of each term, a subsequence of the result is an exhausting sequence, and it satisfies (i) and (ii). One may regard this lemma as the first step in the construction of an "infinite hierarchy", cf. [15], [19].

(3.2) Theorem. Let $M$ be a connected $P^{2}$-irreducible 3-manifold with one end. Suppose $\underline{\pi}_{1}(M, \underline{a}) \rightarrow \pi_{1}(M, \underline{a})$ is an isomorpbism and $M \neq R^{3}$. Then $M=$ $F \times[0, \infty)$ where $F$ is a connected 2-manifold.

Proof. $M$ is end-irreducible so we may select an exhausting sequence $\left\{C_{n}\right\}$ satisfying (i)-(v) of Lemma (3.1). Select a proper map $a:[0, \infty) \rightarrow M$ with $a([n, \infty)) \subset M-C_{n}$. Let $\lambda:\left(S^{1}, *\right) \rightarrow(M, a(0))$ be any loop. Then $\lambda$ defines a map $\mu:\left(\underline{S}^{1}, \underline{*}\right) \rightarrow(M, \underline{a})$ by $\mu \mid \underline{*}=a$, and $\mu \mid S^{1} \times\{n\}$ traces backwards along $a$ from $a(n)$ to $a(0)$, around $\lambda$, then forward along $a$ to $a(n)$. Since $\pi_{1}(M, \underline{a}) \rightarrow \pi_{1}(M, \underline{a})$ is an isomorphism, $\mu$ is homotopic rel $*$ to a proper map. Thus, no matter how large $n$, there is a loop $\nu$ in $M-C_{n}$ based at $a(n)$ so that the isomorphism of $\pi_{1}(M, a(n))$ onto $\pi_{1}(M, a(0))$ defined by $a \mid[0, n]$ carries $[\nu]$ to $[\lambda]$. Thus the inclusion induced homomorphism $\pi_{1}\left(\operatorname{cl}\left(M-C_{n}\right)\right) \rightarrow \pi_{1}(M)$ is an epimorphism. (Note that $M-C_{n}$ is connected by $(v)$ and the fact that $M$ has one end.)

We assert that $\operatorname{Fr}\left(C_{n}\right)$ is connected for every $n$. If not let $F$ and $G$ be distinct components of $\operatorname{Fr}\left(C_{n}\right)$. Since both $C_{n}$ and $M-C_{n}$ are connected, we can choose a loop in $M$ meeting $\operatorname{Fr}\left(C_{n}\right)$ only in $F$ and $G$, and having a nonzero mod 2 intersection number with each of $F$ and $G$. By the above this loop is homotopic to a loop in $M-C_{n}$ which has mod 2 intersection number zero with both $F$ and $G$, a contradiction.

Let $F_{n}=\operatorname{Fr}\left(C_{n}\right)$, a connected 2-sided incompressible surface properly embedded in $M$. Then $\pi_{1}(M)$ is the free product of $\pi_{1}\left(C_{n}\right)$ by $\pi_{1}\left(\operatorname{cl}\left(M-C_{n}\right)\right)$ with amalgamation over $\pi_{1}\left(F_{n}\right)$ (van Kampen's theorem). Since $\pi_{1}\left(\operatorname{cl}\left(M-C_{n}\right)\right) \rightarrow \pi_{1}(M)$ is an epimorphism, $\pi_{1}\left(F_{n}\right) \rightarrow \pi_{1}\left(C_{n}\right)$ is an isomorphism. By (2.1) there is a homomorphism of $F_{n} \times[0, n]$ onto $C_{n}$, with $\left(\partial F_{n}\right) \times[0, n] \cup F_{n} \times\{n\}$ carried to $F_{n}$. Moreover it is not hard to see (e.g., by regular neighborhood theory) that the homeomorphism of $F_{n+1} \times[0, n+1]$ onto $C_{n+1}$ may be chosen to extend that of $F_{n} \times[0, n]$ onto $C_{n}$, and the theorem is proved.

An interesting corollary is a characterization of $R^{3}$. 
(3.3) Let $M$ be an open irreducible contractible 3-manifold with one end. Then $\underline{\pi}_{1}(M, \underline{a})$ is the trivial group iff $M=R^{3}$.

Proof. If $M \neq R^{3}$ then (3.2) applies, but we assume $\partial M \neq \varnothing$. Conversely a loop which misses a pl-ball in $R^{3}$ is contractible in the complement of that ball.

One should note that McMillan [10] has given an uncountable family of open contractible subsets of $R^{3}$, each having just one end.

(3.4) Theorem. Let $M$ be a connected, $P^{2}$-irreducible 3-manifold with more than one end. Then for each end $[a]$ of $M$ the bomomorphism $\underline{\pi}_{1}(M, \underline{a}) \rightarrow \pi_{1}(M, \underline{a})$ is an isomorpbism iff one of (a), (b), or (c) bolds:

(a) $M$ bas two ends and is bomeomorpbic to $F \times R^{1}$ for $F$ a compact 2-manifold.

(b) $M$ bas more than two ends, $\pi_{1}(M)=\{1\}$, and $M$ may be constructed from a cell by removing from the boundary a subset bomeomorpbic to $\pi_{0}(M)$.

(c) $M$ bas more than two ends, $\pi_{1}(M)=Z$, and $M$ may be constructed from the product $D \times S^{1}$ of a disk with $S^{1}$ by removing from the boundary $X \times S^{1}$, where $X$ is a subset of $\partial D$ bomeomorpbic to $\pi_{0}(M)$.

Proof. Select an exhausting sequence $\left\{C_{n}\right\}$ satisfying (i)-(v) of (3.1), and so that $M-C_{n}$ is disconnected for all $n$. If $A_{n}$ is the closure of a component of $M-C_{n}$ then as in (3.2) $\pi_{1}\left(A_{n}\right) \dot{\rightarrow} \pi_{1}(M)$ is an isomorphism. It follows that $F_{n}=$ $A_{n} \cap C_{n}$ is connected and $C_{n}$ is homeomorphic to $F \times[-n, n]$ with $F_{n}$ going to $F \times\{n\}$ and another component of $\operatorname{Fr}\left(C_{n}\right)$ going to $F \times\{-n\}$.

If $M$ has two ends then $\mathrm{cl}\left(C_{n+1}-C_{n}\right)$ has two components. By (2.1) they are homeomorphic to $F \times[n, n+1]$ and $F \times[-(n+1),-n]$. From this it follows that $M$ is homeomorphic to $F \times R^{1}$ and case (a) is proved.

If $M-C_{n}$ has more than two components, then some component $G$ of $\operatorname{Fr}\left(C_{n}\right)$ embeds in $(\partial F) \times(-n, n)$. Since $\pi_{1}(G) \rightarrow \pi_{1}\left(C_{n}\right)$ is an isomorphism, it follows that either $G$ is a disk and $C_{n}$ a cell or $G$ is an annulus and $C_{n}$ is a solid torus. In the first case $\pi_{1}(M)=\{1\}$, in the second case $\pi_{1}(M)=Z$.

Suppose $\pi_{1}(M)=1$, so $C_{n}$ is a cell and each component of $\operatorname{Fr}\left(C_{n}\right)$ is a disk. Let $B$ be the unit ball in $R^{3}$ and $B_{n}$ the concentric ball of radius $(n-1) / n$. Let $b_{n}: C_{n} \rightarrow B$ be an embedding so that

(1) $b_{n}\left(C_{n}\right) \supset B_{n}$,

(2) $b_{n}\left(\operatorname{cl}\left(\partial C_{n}-\operatorname{Fr}\left(C_{n}\right)\right)\right)=\partial B \cap b_{n}\left(C_{n}\right)$,

(3) The total area of $\partial B-b_{n}\left(C_{n}\right)$ is less than $1 / n$.

Let $E$ be a component of $\operatorname{cl}\left(C_{n+1}-C_{n}\right)$. Then $E$ is a cell which meets $C_{n}$ in a component $F$ of $\operatorname{Fr}\left(C_{n}\right)$. Let $E^{1}$ be the component of $\operatorname{cl}\left(B-b_{n}\left(C_{n}\right)\right)$ meeting $b_{n}\left(C_{n}\right)$ in the disk $b_{n}(F)$. Extend $b_{n} \mid F$ to an embedding of $E$ into $E^{1}$. These 
extensions fit together with $b_{n}$ to give an embedding $b_{n+1}$ of $C_{n+1}$ in $B$. They may be chosen so that $b_{n+1}$ satisfies (1)-(3) above with $n+1$ in place of $n$.

Let $b: M \rightarrow B$ be defined by $b \mid C_{n}=b_{n}$. Then $b$ is an embedding of $M$ in $B$. By (1) $b(M) \supset B-\partial B$, by (2) $b(\partial M)=b(M) \cap \partial B$, by (3) $\partial B-b(\partial M)$ is a closed 0 -dimensional set. To see that $\partial B-b(\partial M)$ is homeomorphic to $\underline{\pi}_{0}(M)$, notice that each point of $\partial B-b(\partial M)$ is given as the intersection of a decreasing family of disks. Each of these disks corresponds to a component of $M-C_{n}$, and decreasing intersections of such components are in $1-1$ correspondence with points of $\pi_{0}(M)$. This observation also shows that the topology is correct. This establishes case (b) of the theorem; case (c) is proved similarly.

(3.5) Corollary. Let $M$ be a connected, $P^{2}$-irreducible, end-irreducible, noncompact 3-manifold. Suppose boundary components of $M$ are compact, and for some end $[a]$ of $M, \underline{\pi}_{1}(M, \underline{a}) \rightarrow \pi_{1}(M, \underline{a})$ is an isomorphism. Then for some closed 2-manifold $F, M$ is bomeomorphic to eitber $F \times[0, \infty)$ or to $F \times R^{1}$.

Proof. If $M$ has one end it is homeomorphic to $F \times[0, \infty)$ by (3.2) so we shall assume it has more than one end. Let $\left\{C_{n}\right\}$ be an exhausting sequence satisfying (i)-(v) of (3.1). Let $A_{n}$ be the closure of the component of $M-C_{n}$ which contains [a]. If $F_{n}=A_{n} \cap C_{n}$ then, as in (3.4), $C_{n}$ is homeomorphic to $F_{n} \times[-n, n]$ with $F_{n}$ going to $F_{n} \times\{n\}$. It follows that $M-C_{n}$ has exactly two components, thus $M$ has exactly one other end [b]. Let $B_{n}$ be the closure of the other component of $M-C_{n}$. By (3.2) again $A_{n}$ is homeomorphic to $F_{n} \times[n, \infty)$, from which $\pi_{1}\left(B_{n}\right) \rightarrow \pi_{1}(M)$ is an isomorphism. Since $n$ is arbitrary, $\underline{\pi}_{1}(M, \underline{b}) \rightarrow$ $\pi_{1}(M, \underline{b})$ is an isomorphism. Then (3.4) finishes the corollary.

4. The Waldhausen-Heil theorem.

(4.1) Lemma. Let $F \neq S^{2}$ be a closed connected 2-manifold. Let $p: F \times I \rightarrow$ $N$ be a covering map with $p(F \times\{0\}) \neq p(F \times\{1\})$. Let $G=p(F \times\{0\})$ and let $p_{0}: F \rightarrow G$ be defined by $p_{0}(x)=p(x, 0)$. Then there exist bomeomorphisms $b$ and $b_{1}$ so that the diagram

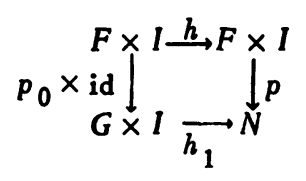

commutes, and $b \mid F \times\{0\}=$ id.

Proof. If $p$ is a homegmorphism, the theorem is trivial. Assume not, then clearly $G$ is a closed connected 2 -manifold not the projective plane, and $N$ is a compact connected 3-manifold. A homotopy cell in $N$ can be lifted into $F \times I$, 
so it is a cell. Since $\partial F=\varnothing, G_{1}=p(F \times\{1\})=\partial N-G$ is a closed nonempty 2manifold. If $p$ is an r-fold covering then $r \chi(G)=\chi(F \times\{0\})=\chi(F \times\{1\})=r \chi(G)$ where $\chi$ is the Euler characteristic.

If $\lambda$ is a loop in $N$ based at a point of $G$, then $\lambda$ can be lifted to a path in $F \times I$ with endpoints in $F \times\{0\}$. A homotopy of this path rel endpoints into $F \times\{0\}$ covers a homotopy of $\lambda$ rel the base point into $G$. Thus $\pi_{1}(G)-\pi_{1}(N)$ is an epimorphism. It follows from (3.4) of [S] that the natural projection $G \times\{0\} \rightarrow$ $G$ can be extended to a homeomorphism $b_{1}$ of $G \times I$ onto $N$.

Let us think of $b_{1}\left(p_{0} \times\right.$ id) as a homotopy of the map $p \mid F \times\{0\}$. Covering this homotopy we obtain a map $b: F \times I \rightarrow F \times I$ so that $b \mid F \times\{0\}=$ id. Since $b^{-1}$ may also be obtained by a covering we have that $b$ is a homeomorphism.

(4.2) Theorem. Let $M$ and $N$ be $P^{2}$-irreducible, boundary-irreducible, and endirreducible connected 3-manifolds. Suppose tbat $M$ is noncompact but tbat boundary components of $N$ are compact. Finally suppose there is a proper map $f:(M, \partial M) \rightarrow$ $(N, \partial N)$ so that $\pi_{1}(f)$ is a monomorpbism. Then there is a proper bomotopy of $f$ (as a map of pairs) to a map $f_{1}$ so that either (a) or (b) bolds.

(a) $f_{1}$ is a finite sheeted covering map.

(b) There are closed 2-manifolds $K$ in $M$ and $L$ in $N$ so that $M$ is a bundle with fibre $R^{1}$ and with zero section $K, f_{1}$ is a covering of $K$ onto $L$, one component of $N-L$ bas closure $L \times[0, \infty)$, and $f_{1}(k, t)=\left(f_{1}(k),|t|\right)$.

Furthermore, if $f \mid \partial M$ is a local bomeomorphism, then the bomotopy may be chosen constant on $\partial M$.

Proof. We observe that since $f$ is proper, components of $\partial M$ are compact and $N$ is not compact. On each boundary component of $M$ we may perform a homotopy of the restriction of $f$ to a covering map ( $M$ is boundary irreducible, $N$ is $P^{2}$. irreducible, and use Theorem 1 of [9]). Using collar neighborhoods of the boundary we may extend this to a homotopy of $f$. Thus we may assume that $f \mid \partial M$ is a covering map.

Choose an exhausting sequence $\left\{C_{n}\right\}$ for $N$ satisfying (i)-(v) of Lemma (3.1). Since $N$ is $P^{2}$-irreducible no component of $\operatorname{Fr}\left(C_{n}\right)$ is a projective plane. No component of $\operatorname{Fr}\left(C_{n}\right)$ is a 2-sphere since $N$ is irreducible and $\operatorname{Fr}\left(C_{n}\right)$ is incompressible. Choose an exhausting sequence $\left\{C_{n}^{\prime}\right\}$ for $M$ satisfying (i)-(v) of (3.1) so that $C_{n}^{\prime} C f^{-1}\left(C_{n}-\operatorname{Fr}\left(C_{n}\right)\right)$, and $f^{-1}\left(C_{n}\right) \subset C_{n+1}^{\prime}-\operatorname{Fr}\left(C_{n+1}^{\prime}\right)$ (we may again need subsequences to achieve this).

Consider the map $f \mid \operatorname{cl}\left(C_{n+1}^{\prime}-C_{n}^{\prime}\right) \rightarrow \operatorname{cl}\left(C_{n+1}-C_{n-1}\right)$. According to Lemma 2 of [9] this map is homotopic rel $\partial\left(\operatorname{cl}\left(C_{n+1}^{\prime}-C_{n}^{n}\right)\right)$ to a map $f_{n}^{\prime}$ which is transverse with respect to $\operatorname{Fr}\left(C_{n}\right)$ and so that $f_{n}^{\prime}-1\left(\operatorname{Fr}_{r}\left(C_{n}\right)\right)_{0}$ is a system of 2-sided imcompressible surfaces in $\mathrm{cl}\left(C_{n+1}^{\prime}-C_{n}^{\prime}\right)$. It follows that $f_{n}^{\prime-1}\left(\operatorname{Fr}\left(C_{n}\right)\right)$ is incompressible in $M$. 
Now $f$ is proper homotopic rel $\partial M$ to a map which agrees with $f_{n}^{\prime}$ on $\operatorname{cl}\left(C_{n+1}^{\prime}-C_{n}^{\prime}\right)$. Thus we may assume that $D_{n}^{\prime}=f^{-1}\left(C_{n}\right)$ is a compact 3-manifold in $M$ with $C_{n}^{\prime} C D_{n}^{\prime}$, and $\partial D_{n}^{\prime}$ incompressible in $M$ (recall $M$ is boundary irreducible, and no component of $\operatorname{Fr}\left(C_{n}\right)$ meets $\left.\partial N\right)$. In particular $\left\{D_{n}^{\prime}\right\}$ is an exhausting sequence for $M$ and no component of $\operatorname{Fr}_{r}\left(D_{n}^{\prime}\right)$ meets $\partial M$. As above no component of $\partial D_{n}^{\prime}$ is a projective plane or a $2-$ sphere.

Applying Theorem 1 of [9] there is a proper homotopy of $f$ rel $\partial M$ to a map $f^{\prime}$ so that $f^{\prime-1}\left(C_{n}\right)=D_{n}^{\prime}$ and if $F$ is a component of $\operatorname{Fr}\left(D_{n}^{\prime}\right)$ then $f^{\prime} \mid F$ is a covering map onto a component of $\operatorname{Fr}\left(C_{n}\right)$. This is so since components of $\operatorname{Fr}\left(C_{n}\right)$, respectively $\operatorname{Fr}\left(D_{n}^{\prime}\right)$, are bicollared in $N$, respectively $M$.

Now let $D$ be a component of $D_{n}^{\prime}$. We may apply Theorem $A$ of [9] to $f^{\prime} \mid D \rightarrow$ $C_{n}$. According to that theorem there are two possibilities:

(a) $f^{\prime} \mid D$ is homotopic rel $\partial D$ to a covering map onto $C_{n}$, or

(b) $D$ is a line bundle over a closed surface and $f^{\prime} \mid D$ is homotopic rel $\partial D=$ $\operatorname{Fr}(D)$ to a map into $\operatorname{Fr}\left(C_{n}\right)$.

Notice that in case (b) there is a homotopy of $f^{\prime}$ constant outside an arbitrarily small neighborhood of $D$ to a map $f_{1}^{\prime}$ with $f_{1}^{\prime-1}\left(C_{n}\right)=D_{n}^{\prime}-D$ and $f_{1}^{\prime-1}\left(C_{n+1}\right)=$ $D_{n+1}^{\prime}$.

By choosing a subsequence of $\left\{C_{n}\right\}$ if necessary the above lead to two cases:

$\left(a_{1}\right)$ There is an exhausting sequence for $M$ whose $n$th term is a component of $D_{n}^{\prime}$ which falls under case (a) above, or

$\left(b_{1}\right)$ given any component of $D_{n}^{\prime}$ there exists $m \geq n$ so that the component of $D_{m}^{\prime}$ containing it falls under case (b).

Applying the homotopy mentioned after case (b) above and choosing a subsequence if necessary we see there is a proper homotopy rel $\partial M$ of $f^{\prime}$ to a map $f_{1}^{\prime}$ so that if $D_{n}=f_{1}^{\prime-1}\left(C_{n}\right)$ then $D_{n}$ consists of some components of $D_{n}^{\prime}$ and either

$\left(a_{2}\right)$ For any $n$ and any component $D$ of $D_{n}, f_{1}^{\prime} \mid D$ is homotopic rel $\partial D$ to a covering map onto $C_{n}$, or

$\left(b_{2}\right) D_{n}$ is a line bundle over a closed connected surface either a product for every $n$ or twisted for every $n$, and $f_{1}^{\prime} \mid D_{n}$ is homotopic rel $\partial D_{n}$ to a map into $\partial C_{n}$.

Let us show that $\left(b_{2}\right)$ leads to case $(b)$ of the present theorem. Under $\left(b_{2}\right)$ clearly $\partial M=\varnothing$. Now $D_{1}$ is a line bundle over a closed surface $K$ and $f_{1}^{\prime} \mid D_{1}$ is homotopic rel $\partial D_{1}$ to a map onto a component of $\operatorname{Fr}\left(C_{1}\right)$. Write a collar neighborhood of this component in $C_{1}$ as $L \times[0,1]$ where the component of $\operatorname{Fr}\left(C_{1}\right)$ is written as $L \times\{1\}$ and $L=L \times\{0\}$ is contained in $C_{1}-\partial C_{1}$. Taking the above homotopy, following it by a strong deformation retraction of $D_{1}$ along the fibres into $K$, and then stretching back along the fibres of $L \times[0,1]$, we see that $f_{1}^{\prime} \mid D_{1}$ 
is homotopic as a map of $\left(D_{1}, \partial D_{1}\right)$ into $\left(C_{1}, L \times\{1\}\right)$ to a map $f_{1} \mid D_{1}$ so that $f_{1}(k) \in L$ for $k \in K$ and $f_{1}(k, t)=\left(f_{1}(k),|t|\right) \in L \times[0,1]$ where each fibre of $D_{1}$ is written $\{k\} \times[-1,1]$ and there is an indeterminancy in the sign.

Using a collar neighborhood of $L \times\{1\}$ in $\mathrm{cl}\left(N-C_{1}\right)$ we see there is a proper homotopy of $f_{1}^{\prime}$ to a map $g_{1}$ so that $g_{1}\left|D_{1}=f_{1}\right| D_{1}$ and $g_{1} \mid \operatorname{cl}\left(M-D_{2}\right)=$ $f_{1}^{\prime} \mid \operatorname{cl}\left(M-D_{2}\right)$. Now let $S$ be a component of $\operatorname{cl}\left(D_{2}-D_{1}\right)$. Then $\partial S$ meets both $\partial D_{1}$ and $\partial D_{2}$ (recall $D_{2}$ is a line bundle too and is twisted iff $D_{1}$ is twisted). Let $F$ and $F^{\prime}$ be components of $\partial S \cap \partial D_{1}$ and $\partial S \cap \partial D_{2}$ respectively. Let $l$ be a loop on $F$. Twice around $l$ is homotopic in $S$ to a loop in $F^{\prime}$ since $\pi_{1}\left(F^{\prime}\right)$ is of index one or two in $\pi_{1}\left(D_{2}\right)$ and $\pi_{1}(S) \rightarrow \pi_{1}\left(D_{2}\right)$ is a monomorphism. It follows from Proposition 5 of [9] that $S$ is homeomorphic to $F \times I$. Then by Theorem $A$ of [9], $g_{1} \mid S \rightarrow\left(C_{2}-C_{1}\right)$ is homotopic rel $\partial S$ to a covering map $f_{1} \mid S$ onto a component $T$ of $\operatorname{cl}\left(C_{2}-C_{1}\right)$. By Lemma (4.1) we may write $S=F \times[1,2], T=$ $f_{1}(F) \times[1,2]=L \times[1,2]$, and $f_{1}(s, t)=\left(f_{1}(s), t\right)$. If $\operatorname{cl}\left(D_{2}-D_{1}\right)$ has another component we may proceed similarly. Notice that $D_{2}$ is the same line bundle as $D_{1}$. We use the constructed product line bundle structures on the components of $\operatorname{cl}\left(D_{2}-D_{1}\right)$ to extend our line bundle structure to $D_{2}$.

There is a proper homotopy of $g_{1}$, constant on $D_{1} \cup \mathrm{cl}\left(M-D_{2}\right)$ to a map $g_{2}$ so that $g_{2}\left|\operatorname{cl}\left(D_{2}-D_{1}\right)=f_{1}\right| \operatorname{cl}\left(D_{2}-D_{1}\right)$. Notice we have defined the desired map $f_{1}$ on $D_{2}$, and have begun the construction of the proper homotopy. Continuing inductively we get the structure of a line bundle over $K$ on $D_{n}$ with a metric which gives every fibre a radius $n$, a proper map $g_{n}: M \rightarrow N$ homotopic rel $D_{n-1} \cup \mathrm{cl}\left(M-D_{n}\right)$ to $g_{n-1}$, a component of $C_{n}-L$ with closure $L \times[0, n]$ and so that $g_{n}(k, t)=\left(f_{1}(k),|t|\right)$. If $f_{1}: N \rightarrow M$ is defined by $f_{1}\left|D_{n}=g_{n}\right| D_{n}$, then $f_{1}$ is as desired in case (b) of the theorem.

Now suppose case $\left(\mathrm{a}_{2}\right)$ above holds. Consider a component $S$ of $\operatorname{cl}\left(D_{n+1}-D_{n}\right)$. Apply Theorem $A$ of [0] to $f_{1}^{\prime} \mid S \rightarrow \operatorname{cl}\left(C_{n+1}-C_{n}\right)$. If, for every $n$ and every $S$, $f_{1}^{\prime} \mid S$ is homotopic rel $\partial S$ to a covering map, then these homotopies fit together to give a proper homotopy constant on $\partial M$ to a covering map $f: M \rightarrow N$, proving case (a) of the theorem. Suppose then that $n(>1)$ is chosen smallest so that $S$ is a line bundle over a closed surface and $f_{1}^{\prime} \mid S$ is homotopic rel $\partial S$ to a map into $f_{1}^{\prime}(\partial S)$. Then $\partial S \subset \operatorname{Fr}\left(D_{n}\right)$, since if $\partial S \subset \operatorname{Fr}\left(D_{n+1}\right)$ then $S$ is a component of $D_{n+1}$ and this is ruled out under $\left(a_{2}\right)$. Then there is a homotopy of $f_{1}^{\prime}$, constant outside a small neighborhood of $S$ to a map $g_{1}$ so that $g_{1}^{-1}\left(C_{n+1}\right)=D_{n+1}$ and $g_{1}^{-1}\left(C_{n}\right)=$ $D_{n} \cup S$. Notice that $\operatorname{cl}\left(D_{n+1}-\left(D_{n} \cup S\right)\right)=\operatorname{cl}\left(D_{n+1}-D_{n}\right)-S$, in particular the number of components has decreased.

It may now be that the restriction $g_{1} \mid D$ of $g_{1}$ to the component $D$ of $g_{1}^{-1}\left(C_{n}\right)$ containing $S$ is no longer homotopic rel $\partial D$ to a covering map. We may now do a homotopy of $g_{1}$, constant outside a small neighborhood of $D$, to a map $g_{2}$ so that 
$g_{2}(D) \subset C_{n+1}-C_{n}$. We are now back in case $\left(a_{2}\right)$ since $g_{2}^{-1}\left(C_{n+1}\right)=D_{n+1}$. Moreover $g_{2}^{-1}\left(C_{n}\right)-g_{2}^{-1}\left(C_{n-1}\right)$ has fewer components than $g_{1}^{-1}\left(C_{n}\right)-g_{1}^{-1}\left(C_{n-1}\right)$, which has at most as many components as $D_{n}-D_{n-1}$ (this last depends on whether $S$ is twisted or not).

On the other hand it may happen that $g_{1}$ already satisfies $\left(a_{2}\right)$, but the component of $\operatorname{cl}\left(g_{1}^{-1}\left(C_{n}\right)-D_{n-1}\right)$ which contains $S$ has all its boundary in $\partial D_{n-1}$, is again a line bundle, and the restriction of $g_{1}$ is homotopic to a map into $\partial C_{n-1}$ (that is, $n$ is no longer smallest). We perform a homotopy as we did on $f_{1}^{\prime}$ this time decreasing the number of components in $\operatorname{cl}\left(g_{1}^{-1}\left(C_{n}\right)-D_{n-1}\right)$. After a finite number of steps we have constructed a map $g_{2}$, proper homotopic rel $\partial M$ to $g_{1}$ by a homotopy which is constant outside $D_{n+1}$, so that $g_{2}$ is again in case $\left(a_{2}\right)$. Moreover if $g_{2}^{-1}\left(C_{k}\right) \neq D_{k}$ for some $k \leq n$ then $g_{2}^{-1}\left(C_{k}\right)-g_{2}^{-1}\left(C_{k-1}\right)$ has fewer components than $D_{k}-D_{k-1}$, while if $g_{2}^{-1}\left(C_{k}\right)=D_{k}$ for some $k \leq n$ then the homotopy is constant on $D_{k}$. Finally, $g_{2} \mid S$ is homotopic rel $\partial S$ to a covering map if $S$ is a component of $\mathrm{cl}\left(g_{2}^{-1}\left(C_{k}\right)-g_{2}^{-1}\left(C_{k-1}\right)\right)$ and $k \leq n$.

We now proceed by induction to improve $g_{2}$. Since the number of components of $\operatorname{cl}\left(D_{k}-D_{k-1}\right)$ cannot decrease indefinitely, the process stabilizes on the inverse image of $C_{k}$ after a finite number of steps. We thus construct a proper homotopy of $f_{1}^{\prime}$ rel $\partial M$ to a map of the type described at the beginning of this case. This establishes case (a) and the theorem.

(4.3) Definition. Let $M$ and $N$ be connected 3-manifolds, $M$ having a finite number of compact boundary components and a finite number of ends. A homomorphism $\psi: \pi_{1}(M) \rightarrow \pi_{1}(N)$ is said to respect the peripberal structure provided:

(i) If $F$ is a boundary component of $M$ then there is a boundary component $G$ of $N$ so that $\psi i_{*}\left(\pi_{1}(F)\right)$ is conjugate in $\pi_{1}(N)$ to a subgroup of $i_{*}\left(\pi_{1}(G)\right.$ ). (Here $i_{*}$ denotes the homomorphism induced by inclusion followed by change of base point along a path. The definition does not depend on the choice of path.)

(ii) For any end [a] of $M$ there exists an end [b] of $N$ so that $\psi^{\infty} i_{*}\left(\underline{\pi}_{1}(M, \underline{a})\right)$ is conjugate to a subgroup of $i_{*}\left(\underline{\pi}_{1}(N, \underline{b})\right)$ by an element of $\pi_{1}(N, \underline{b})$ which is a single element of $\pi_{1}(N)$ with base point shifted along $b$ to each $b(n)$. (Here $\psi^{\infty}: \pi_{1}(M, \underline{a}) \rightarrow \pi_{1}(N, \underline{b})$ is the homomorphism induced by the sequence $\psi_{n}: \pi_{1}(M, a(n)) \rightarrow \pi_{1}(N, b(n))$, where $\psi_{n}$ is $\psi$ with base points shifted along $a$ and $b$. Again $i_{*}$ represents the natural inclusion homomorphism.) The definition is independent of the choice of representatives $a$ and $b$ of $[a]$ and [b].

(4.4) Theorem. Let $M$ and $N$ be connected noncompact 3-manifolds, $M$ baving a finite number of compact boundary components and a finite number of ends. Suppose $N$ is $P^{2}$-irreducible, boundary-irreducible, and end-irreducible. Let $\psi: \pi_{1}(M) \rightarrow \pi_{1}(N)$ be a bomomorphism. Then there exists a proper map 
$f:(M, \partial M) \rightarrow(N, \partial N)$ wbich induces $\psi$ iff $\psi$ respects the peripheral structure.

Proof. The necessity is easy, we prove sufficiency. If we can construct a proper map $f: M \rightarrow N$ which induces $\psi$, then the argument in (6.3) of [15] finishes the proof. First select a maximal tree $T$ in $M$ with just one end for each end of $M$. That this can be done may be seen as follows. Select a maximal tree $T^{\prime}$ and an exhausting sequence $\left\{C_{n}\right\}$ for $M$ of connected subcomplexes so that $T^{\prime} \cap C_{n}$ is connected, and components of $T^{\prime}-C_{n}$ are unbounded for every $n$. Suppose for some $n_{0}$ that if $A_{n_{0}}$ is an unbounded component of $M-C_{n_{0}}$, then $A_{n_{0}} \cap T^{\prime}$ has just one (unbounded) component. Now let $A_{n_{0+1}}$ be an unbounded component of $M-C_{n_{0+1}}$. Then any component of $T^{\prime} \cap A_{n_{0+1}}$ has exactly one 1-simplex with a vertex in $C_{n_{0+1}}$ since $T^{\prime} \cap C_{n_{0+1}}$ is connected and $T^{\prime}$ is a tree. If there is more than one unbounded component in $T^{\prime} \cap A_{n_{0+1}}$ then some 1-simplex of $A_{n_{0+1}}$ joins two such. We add it to $T^{\prime}$ and subtract one of the 1 -simplexes with a vertex in $C_{n 0+1}$. This procedure produces a maximal tree of the desired type, even if $M$ has infinitely many ends.

We define $f: T \rightarrow N$ first. Choose $n$ large enough that each unbounded component of $M-C_{n}$ determines just one end of M. Map $C_{n} \cap T$ and all bounded components of $T-C_{n}$ to the base point of $N$. For each end [a] of $M$ let $a:[0, \infty) \rightarrow T$ be the unique one-to-one simplicial map representing $[a]$ and so that $a(0)$ is the base point of $M$. Let $b$ represent the corresponding end of $N$ (see (ii) of (4.3)), and let $\lambda$ be the constant loop by which we must conjugate. If $\sigma$ is the unique 1-simplex of $\operatorname{im}(a)$ with one vertex in $C_{n}$ and the other in $M-C_{n}$, we let $f$ loop $\sigma$ around $\lambda$ and then continue along $b$ so that $f(a(m))=b(m)$ for $m \gg n$. By this we have mapped a subtree $T_{1}$ of $T$, with the property that components of $T-T_{1}$ are bounded, and have closure meeting $T_{1}$ in a single vertex. Map the whole component to the image of the vertex. This defines $f$ as a proper map on $T$.

If $\sigma$ is a 1 -simplex of $M-T$ then $\sigma$ together with $T$ determine a loop on $M$. The endpoints of $\sigma$ have been mapped, $\psi$ specifies a homotopy class of paths joining these points to which $\sigma$ should be mapped. Let $f$ map $\sigma$ to one such path missing as many terms as possible of some fixed exhausting sequence for $N$. We show that since $\psi$ preserves the peripheral structure, this extension of $f$ to the 1 skeleton of $M$ is proper.

If not there is a sequence of 1-simplexes $\left\{\sigma_{n}\right\}$ of $M-T$ converging to the end $[a]$ of $M$, and a compact set $C$ in $N$ so that $f\left(\sigma_{n}\right) \cap C \neq \varnothing$. Moreover, if $\lambda_{n}$ is the loop based at $a(0)$ and determined by $T$ and $\sigma_{n}$ then for any extension of $f$ over $\sigma_{n}$ so that $\left[f \lambda_{n}\right]=\psi\left[\lambda_{n}\right]$ we must have that $f\left(\sigma_{n}\right) \cap C \neq \varnothing$. Let $\mu_{n}$ be the loop based at $a(n)$ and determined by $T$ and $\sigma_{n}$. Then $(a(0) a(n))\left(\mu_{n}\right)(a(n) a(0))$ is homotopic rel $a(0)$ to $\lambda_{n}$ where $a(0) a(n)$ means the path in $T$ from $a(0)$ to $a(n)$. 
Since $T$ has only one end at the end $[a]$ of $M$, it follows that the sequence $\left\{\mu_{n}\right\}$ converges to $[a]$. Thus $\left\{\mu_{n}\right\}$, together with the map $a$, determines an element $[\mu]$ of $\underline{\pi}_{1}(M, \underline{a})$. Now $\underline{b}=f a$ and we have extended $f$ so that $[f \mu]=i_{*}[\nu]$, where [v] $\epsilon_{\underline{\pi}_{1}}(N, \underline{b})$, and the equality holds in $\pi_{1}(N, \underline{b})$. But then for $n \gg 1, f \mu_{n}$ is homotopic rel $f(a(n))=b(n)$ to $\nu_{n}=\nu \mid S^{1} \times n$, and $\nu_{n} \cap C=\varnothing$. Then we could have chosen $f \mid \sigma_{n}$ to be the path: $\left(f\left(\sigma_{n}(0) a(n)\right)\right)\left(\nu_{n}\right)\left(f\left(a(n) \sigma_{n}(1)\right)\right)$, where $\sigma_{n}(0)$ is the initial point of $\sigma_{n}$, and $f\left(\sigma_{n}(0) a(n)\right)$ is the composition of $f$ with a path in $T$. For $n \gg 1$ this misses $C$ since $f \mid T$ is proper.

Since $\psi$ is a homomorphism, we can extend $f$ over the 2-skeleton of $M$, we do so again missing as many terms of our exhausting sequences as we can. Since $N$ is end-irreducible, this extension is proper also. Since $N$ is $P^{2}$-irreducible we can extend $f$ over the 3-skeleton of $M$, and we do so in the same way as above. Since $N$ is end-irreducible, in particular $N \neq R^{3}$, this extension is proper also.

We give the corresponding theorem now for the case where $M$ has infinitely many ends and/or infinitely many compact boundary components. The definition of "respects the peripheral structure" is somewhat more complicated since we must be careful to respect the nontrivial topology of $\pi_{0^{\circ}}$.

(4.5) Definition. Let $M$ and $N$ be connected 3-manifolds, $M$ having compact boundary components. A homomorphism $\psi: \pi_{1}(M) \rightarrow \pi_{1}(N)$ is said to respect the peripheral structure provided:

(i) For each end $[a]$ of $M$ there exists an end $[b]=f_{0}([a])$ of $N$ so that $\psi^{\infty} i_{*}\left(\pi_{1}(M, \underline{a})\right)$ is conjugate to a subgroup of $i_{*}\left(\pi_{1}(N, \underline{b})\right)$ by an element of $\pi_{1}(N)$ as in (ii) of (4.3). The map $\underline{f}_{0}: \underline{\pi}_{0}(M) \rightarrow \underline{\pi}_{0}(N)$ is continuous. Further, if $\alpha: T \rightarrow M, \beta: T \rightarrow N$ are proper maps of a tree $T$ so that $\underline{L}_{0} \underline{\pi}_{0}(\alpha)=\underline{\pi}_{0}(\beta)$, then there exists a compact set $C \subset T$ so that if $[a]$ and $\left[a^{\prime}\right]$ are ends of $T$ in the same component of $T-C$, then $\psi^{\infty} i_{*}\left(\pi_{1}(M, \underline{a a})\right)$ and $\psi^{\infty} i_{*}\left(\pi_{1}\left(M, \underline{a} \underline{a}^{\prime}\right)\right)$ are conjugate to subgroups of $i_{*} \underline{I}_{1}(N, \underline{\beta} \underline{a})$ and $i_{*} \Pi_{1}\left(N, \underline{\beta} \underline{a}^{\prime}\right)$ respectively by the same element of $\pi_{1}(N)$. (Here $a$ and $a^{\top}$ are the unique simple simplicial maps of $[0, \infty) \rightarrow T$ representing $[a]$ and $\left[a^{\prime}\right]_{0}$ )

(ii) If $F$ is a boundary surface of $M$, there exists a boundary surface $G=f_{0}(F)$ of $N$ so that $\psi i_{*}\left(\pi_{1}(F)\right)$ is conjugate to a subgroup of $i_{*}\left(\pi_{1}(G)\right)$ by an element of $\pi_{1}(N)$. The map $f_{0}$ is consistent with $f_{0}$ in the sense that if a sequence $\left\{F_{n}\right\}$ in $\pi_{0}(\partial M)$ converges to the end $[a]$ of $M$, then $\left\{f_{0}\left(F_{n}\right)\right\}$ converges to the end $[b]^{n}=$ $L_{0}([a])$. Also if the conjugating elements of $\pi_{1}(N)$ for $\left\{F_{n}\right\}$ have base points shifted along $b$ to $b(n)$, then conjugating the element of $\pi_{1}(N, \underline{b})$ so defined by the element of $\pi_{1}(N)$ which carries $\psi^{\infty} i_{*}\left(\underline{\pi}_{1}(M, \underline{a})\right)$ into $i_{*}\left(\pi_{1}(N, \underline{b})\right)$, also carries this element into $i_{*}\left(\pi_{1}(N, \underline{b})\right)$.

(4.6) Theorem. Let $M$ be a connected 3-manifold witb only compact boundary 
components. Let $N$ be a $P^{2}$-irreducible, boundary-irreducible, and end-irreducible connected 3-manifold. Let $\psi: \pi_{1}(M) \rightarrow \pi_{1}(N)$ be a bomomorphism. There exists a proper map $f:(M, \partial M) \rightarrow(N, \partial N)$ which induces $\psi$ iff $\psi$ respects the peripheral structure.

Proof. Again the necessity is not hard and we only prove sufficiency. If $T$ is a maximal tree in $M$ with one end for each end of $M$, then there is a proper map $T \rightarrow N$ realizing $\underline{L}_{0}$ since $\underline{I}_{0}$ is continuous. The part of $(i)$ in (4.5) about trees enables one to construct the appropriate $f: T \rightarrow N$ by looping around a finite number of members of $\pi_{1}(N)$. The extension of $f$ to a proper map $M \rightarrow N$ proceeds as in (4.4).

The first step in the construction of the homotopy of $f(F)$ to $G=f_{0}(F)$ is to choose a path joining them in $N$. That the paths may be chosen properly (i.e. so the homotopy will be proper) is the consistency part of (ii) in (4.5). One then throws a loop in each path so that $\psi i_{*}\left(\pi_{1}(F)\right) \subset i_{*}\left(\pi_{1}(G)\right)$. That this may be done as to keep the paths proper is implied by the last sentence of (ii). Construct the homotopies missing as many terms of an exhausting sequence as one can, and the resulting homotopy of all boundary components will be a proper map.

(4.7) Theorem. Let $M$ and $N$ be noncompact connected 3-manifolds. Suppose that $M$ and $N$ are $P^{2}$-irreducible, boundary-irreducible, and end-irreducible, and bave only compact boundary components. Let $\psi: \pi_{1}(M) \rightarrow \pi_{1}(N)$ be an isomorphism which respects the peripheral structure. Then either (a), (b), or (c) bolds:

(a) $M$ is bomeomorpbic to $N$ by a bomeomorpbism which induces $\psi$.

(b) $M$ is a vector bundle over a closed surface $K, N$ is bomeomorphic to $L \times[0, \infty)$, there is a proper map $f: M \rightarrow N$ inducing $\psi$, mapping $K$ bomeomorpbically onto $L$, and $f_{1}(k, t)=\left(f_{1}(k),|t|\right)$ with respect to a metric on $M$.

(c) $M$ is a twisted vector bundle over a closed surface $K, N$ is bomeomorphic to $L \times R^{1}$, there is a proper map $f_{1}: M \rightarrow N$ inducing $\psi$, mapping $K$ bomeomorpbically onto $L$, and $f_{1}(k, t)=\left(f_{1}(k),|t|\right)$, with respect to a metric on $M$.

Proof. By (4.6) there exists a proper map $f:(M, \partial M) \rightarrow(N, \partial N)$ inducing $\psi$. We apply (4.2) to the map $f_{\text {. If }} f_{1}$ is a covering map, then it is one sheeted and we have case (a) above. Suppose $f_{1}$ satisfies (b) of (4.2). Then $M$ is a vector bundle over a closed 2-manifold $K$, and $f_{1} \mid K$ is a homeomorphism onto $L$. Applying (3.5) to $N$ we conclude either $N$ is homeomorphic to $L \times[0, \infty)$, or to $L \times R^{1}$. In the first possibility we have case (b) of the present theorem, in the second we either have case (c) or else $M$ is homeomorphic to $K \times R^{1}$. We are then back in case (a). Notice that (a), (b), and (c) are exclusive, and every one actually occurs.

5. Examples. We would like to give a few examples of manifolds to which Theorem (4.7) applies. One way to get a $P^{2}$-irreducible 3 -manifold is to remove 
from $S^{3}$ a closed set $X$ which cannot be separated by a 2-sphere in $S^{3}$. One way to make $S^{3}-X$ end-irreducible is to construct $X$ as the intersection of a decreasing sequence $S^{3}=W_{0} \supset W_{1} \supset \ldots$ of compact 3 -manifolds tamely embedded in $s^{3}$ and so that $\partial W_{n}$ is incompressible in $\left(W_{n-1}-W_{n}\right)$.

Example 1. Antoine's necklace with knots. A typical construction of Antoine's necklace is pictured in Figure 1.

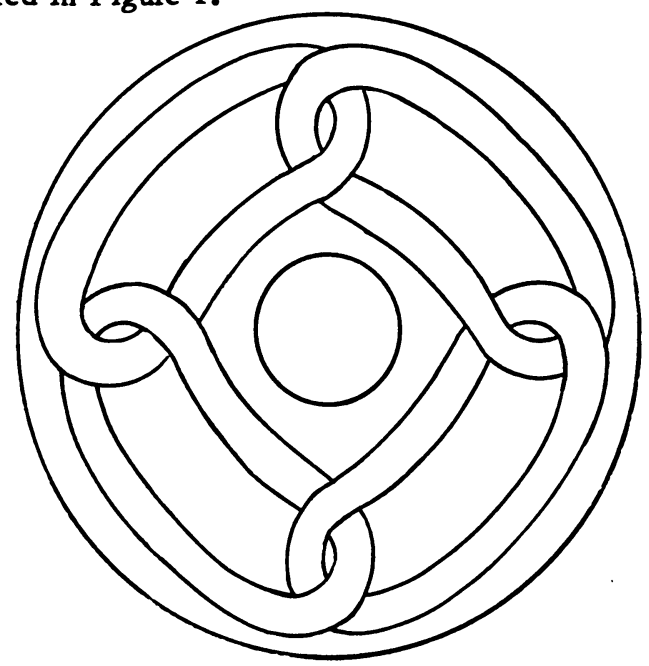

Figure 1

$w_{1}$ is the four linked solid tori. Inside each of the components of $W_{1}$ are four more solid tori linked around the component in the same way that $W_{1}$ is linked around the large solid torus pictured. These sixteen solid tori make up $W_{2}$, etc. There is nothing special about the number four, or about the fact that each torus was drawn unknotted. These may be varied from stage to stage of the construction. The important things are that the tori should be linked and that the diameters of successive components should decrease to zero.

If $X=\bigcap_{n} W_{n}$, then $X$ is compact and zero dimensional. Indeed $X \cong \mathbb{\pi}_{0}\left(S^{3}-X\right)$. The open 3-manifold $S^{3}-X$ is $P^{2}$-irreducible and end-irreducible. If $Y$ is another compact zero dimensional set in $S^{3}$, then a homeomorphism $f$ of $S^{3}-X$ onto $S^{3}-Y$ may be extended to a homeomorphism of the pair $\left(S^{3}, X\right)$ onto the pair $\left(S^{3}, Y\right)$. The reader may check that the above construction provides an uncountable family of distinct examples.

Example 2. Some wild knots. Let $k$ be a knot in $S^{3}$ with the following property: Given any $\epsilon>0$, there are a finite number of disjoint cells $B_{1}, \cdots, B_{n}$ tamely embedded in $S^{3}$, so that the diameter of each $B_{i}$ is less than $\epsilon$, so that $k \cap \partial B_{i}$ is two points, and so that $k \cap \mathrm{cl}\left(S^{3}-\cup_{B_{i}}\right)$ is tame. Then $S^{3}-k$ is $P^{2}$-irreducible and has one irreducible end. This is an uncountable class of knots including all tame knots.

If $k$ is any knot in $S^{3}$, then $S^{3}-k$ is $P^{2}$-irreducible. We do not have an example of a knot $k$ so that $S^{3}-k$ fails to be end-irreducible. 


\section{BIBLIOGRAPHY}

1. R. Bing, Locally tame sets are tame, Ann. of Math. (2) 59 (1954), 145-158. MR 15, 816.

2. W. Browder, J. Levine and G. Livesay, Finding a boundary for an open manifold, Amer. J. Math. 87 (1965), 1017-1028. MR 32 \#6973.

3. E. M. Brown, The Hauptvermutung for 3-complexes, Trans. Amer. Math. Soc. 144 (1969), 173-196. . MR $40 \# 4956$.

4. - On proper homotopy type, Lecture Notes in Math., vol. 375, SpringerVerlag, Berlin and New York.

5. —, Unknotting in $M^{2} \times I$, Trans. Amer. Math. Soc. 123 (1966), 480-505.

6. C. H. Edwards, Jr., Open 3-manifolds which are simply-connected at infinity, Proc. Amer. Math. Soc. 14 (1963), 391-395. MR 27 \#732.

7. H. Freudenthal, Über die Enden topologischer Räume und Gruppen, Math. Z. 33 (1931), 692-713.

8. L. S. Husch and T. M. Price, Finding a boundary for a 3-manifold, Ann. of Math. (2) 91 (1970), 223-235. MR 41 \#9269.

9. W. Heil, On $P^{2}$-irreducible 3-manifold, Bull. Amer. Math. Soc. 75 (1969) 772-775. MR $40 \# 4958$.

10. D. R. McMillan, Jro, Some contractible open 3-manifolds, Trans. Amer. Math. Soc. 102 (1962), 373-382. MR 25 \#561.

11. E. Moise, Affine structures in 3-manifolds. VIII. Invariance of the knot-types; tocal tame imbedding, Ann. of Math. (2) 59 (1954), 159-170. MR 15 \#889.

12. L. C. Siebenmann, Infinite simple homotopy types, Indag. Math. 32 (1970), 479495.

13. $\longrightarrow$ On detecting open collars, Trans. Amer. Math. Soc. 142 (1969), 201-227. MR 39 \#7605.

14. - The obstruction to finding a boundary for an open manifold of dimension greater than 5, Thesis, Princeton University, Princeton, N. J., 1965.

15. F. Waldhausen, On irreducible 3-manifolds which are sufficiently large, Ann. of Math. (2) 87 (1968), 56-88. MR 36 \#7146.

16. J. H. C. Whitehead, Simplicial spaces, nuclei, and m-groups, Proc. London Math. Soc. 45 (1939), 243-327.

17. E. C. Zeeman, Seminar on combinatorial topology, Inst. Hautes Etudes Sci., Paris, 1963. (mimeographed)

18. E. M. Brown and R. H. Crowell, Deformation retractions of 3-manifolds into their boundaries, Ann. of Math. (2) 82 (1965), 445-458. MR 31 \#6236.

19. M. S. Brown, Constructing isotopies in noncompact 3-manifolds, Bull. Amer. Math. Soc. 78 (1972), 461-464.

DEPARTMENT OF MATHEMATICS, DARTMOUTH COLLEGE, HANOVER, NEW HAMPSHIRE 03755

DEPARTMENT OF MATHEMATICS, PRINCETON UNIVERSITY, PRINCETON, NEW JERSEY 08540

Current address (T. W. Tucker): Department of Mathematics, Colgate University, Hamilton, New York 13346 